\title{
Landmark-based Matching Algorithm for Cooperative Mapping by Autonomous Robots
}

\author{
Göksel Dedeoglu and Gaurav S. Sukhatme \\ Robotics Research Laboratory \\ Computer Science Department \\ University of Southern California \\ Los Angeles, CA 90089-0781 \\ dedeoglu,gaurav@ robotics.usc.edu
}

\begin{abstract}
This paper describes a landmark-based algorithm for map matching, and demonstrates its use in efficiently combining topological maps of indoor environments built by autonomous mobile robots. Results of the first implementation are presented, in which two robots with different mechanics and sensory capabilities independently explore their environments with no a priori maps. Each robot is initially unaware of the other's relative position and orientation. Using the match algorithm, they eventually merge their maps into a topologically correct single map in real time, based only on the feature sets they have discovered independently.
\end{abstract}

\section{Introduction}

As autonomous mobile robots are increasingly expected to share dynamic, un-instrumented, indoor spaces with humans and other robots, purposeful navigation and mapping capabilities arise as key requirements [2]. Broadly speaking, research efforts have led to two distinct mapping techniques: Metric and topological maps. Metric approaches [5] are typically implemented as occupancy grids, first introduced by [10] and [7], and later used by [13,14]. On the other hand, topological maps view the world as a collection of interconnected landmarks $[8,9,4,1]$, often augmented with distance information and defined using probability distributions [3,12,11].

Our research addresses the development of algorithms for distributed, anytime, topological map building that can efficiently scale to groups of heterogeneous robots. This paper builds upon our existing mapping strategy [6], and demonstrates a simple, yet robust starting point for the task of cooperative mapping with multiple robots. An algorithm for combining two topological maps is evaluated, and preliminary results with two robots are shown.

Section 2 highlights the topological map components that underly the algorithm, which, in turn, is described in Section 3. A number of map combination experiments are reported in Section 4, providing evidence for the feasibility of the approach. 


\section{Mapping System}

The mapping and navigation algorithms used in this work have been described in detail in [6]. The two mobile robots used in this study are Pioneer 1-AT (Vespucci) and Pioneer 2-DX (Milka) platforms with different kinematics and sensory equipment. Vespucci uses a gyroscope to enhance its position tracking, and uses a Pan-Tilt-Zoom camera connected to the FastTrack vision system to detect visual color blobs. In contrast to Vespucci which has only seven sonar transducers, Milka has sixteen, and carries a SICK laser range finder as well.

The Building blocks of our topological mapping scheme are corners, Tjunctions, end-of-corridors and closed doors, and links interconnecting them, as shown in Figure 1. Based on the perception of these features, each robot is capable of generating and updating a topological map augmented with metric information [6], and refer back to it for enhancing its estimated position.

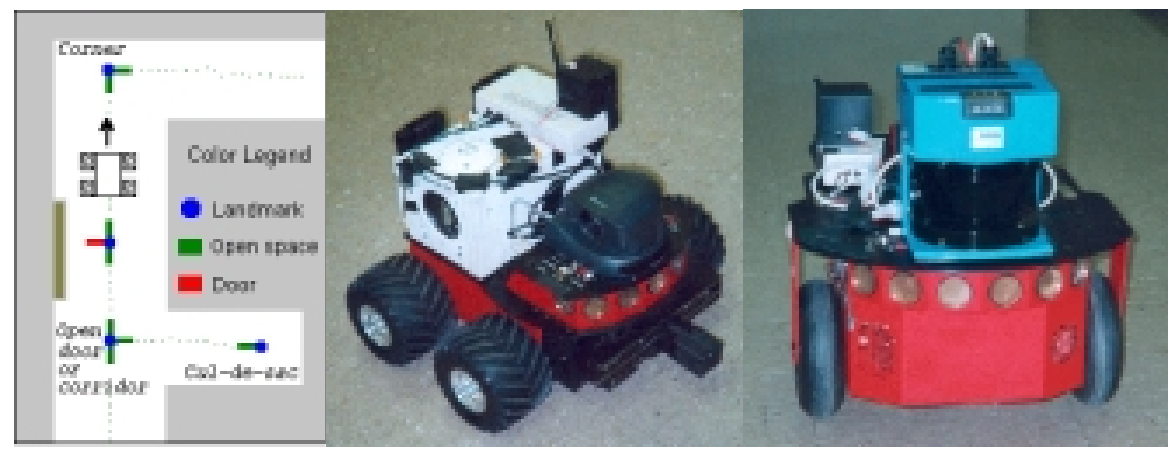

Fig. 1. On the left, topological landmarks and their attributes are illustrated on a sample map. Robots Vespucci (middle) and Milka (right) are shown.

Both Vespucci and Milka use their sonar arrays to detect corridors, openings that can be either open doors or hallways, junctions, and end-of-corridors. While Vespucci seeks for color blobs for detecting closed doors, Milka looks for signatures in laser scans for the same purpose.

As reported in [6], over a set of 300 sonar-based feature detection cases, the rate for correct detection was found to be $81 \%$, along with $20 \%$ of false alarms. For closed door detection, the color-blob detector had a success of $92 \%$ correct detection with $3 \%$ false alarm rate over 180 cases. Laser scanner-based door detector performed at a correct detection rate of $85 \%$ over 130 cases. The associated signal processing and basic feature detection is described in [6] as is the algorithm for single robot mapping and navigation. 


\subsection{Data Structures}

Our map is a collection of landmark elements that are the nodes of an augmented graph. A node carries the following attributes:

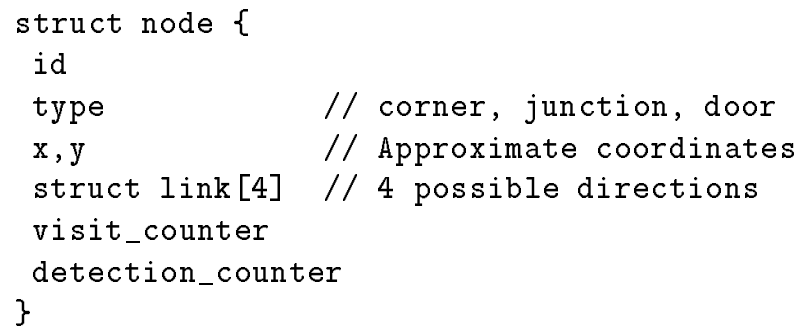

A variation of landmark types can be obtained based on the link structure's type attribute. A corner has two consecutive neighboring links as blocked, whereas the others will be open. An end-of-corridor has only one link that is open, which connects it to other nodes.

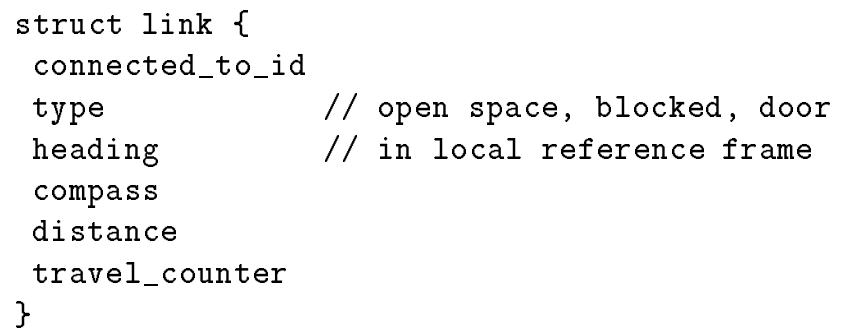

\section{Map-Matching Algorithm}

\subsection{Heuristics}

The goal of the map matching algorithm is to find the transformation $T_{12}$ that relates a robot's $\left(R_{1}\right)$ coordinate system with that of another $\left(R_{2}\right)$, thus enabling the merging of the two maps. The basic intuition is that, if the robots have visited at least partially overlapping spaces and registered enough of the same features in their maps, it should be possible to find and measure the correlation between their maps. Using this idea, the search space of our algorithm is the set of all landmark pairs $\left(L_{R 1}, L_{R 2}\right)$, since each landmark discovered by $R_{1}$ could potentially correspond to any other reported by $R_{2}$. Furthermore, since the landmarks carry heading and position information, each pair defines a unique match, called a candidate transformation. Our algorithm uses two important heuristics to reduce this search space.

H1 Only landmarks of the same type attribute are paired up (e.g., doors with doors, corners with corners)

H2 Only landmarks that describe spatial features (i.e. T-junctions, corners and end of corridors) are considered as candidates. 
The reasoning behind $\mathbf{H 1}$ is that, over the period of time in which the robots build and maintain their maps, a relatively small number of features change their attribute (open vs. closed door) or disappear totally. In other words, the heuristic assumes that the dynamics of the environment are slow relative to the speed of exploration. In addition, H2 cuts the search space down further by relying on those features which are not only less prone to false alarms, but also represent an inherently sparser set in typical indoor environments (e.g., merely a handful of corners and T-junctions as opposed to dozens of doors on a given floor).

\subsection{Map Matching}

The preprocessing step applies the heuristics discussed above and produces a reduced search space, populated by the so-called selected pairs of landmarks. At this stage, these pairs are further tested for possible mismatches in attributes that reflect absolute measurements. When available, compass readings are used to match approximately (+/- 30 degrees), or the candidate is eliminated. In case the landmarks were registered while the robots traveled in opposite directions, a hypothesized compass value is used for comparison.

Finally, for all remaining candidate transformations, translation and rotation components are computed by requiring both the coordinates and the arm-type attributes of the two landmarks $\left(L_{R 1}, L_{R 2}\right)$ to coincide. The latter is then evaluated by applying it to all elements of $R_{2}$ 's map, and counting the number of overlapping and matching landmarks. Having tried all transformations, the one that yields the highest number of match-counts is selected, and the resulting combined map is presented to the user as the best match. In the next section, we analyze 26 such match trials and evaluate the matching algorithm.

\section{Experimental Evaluation}

\subsection{USC Salvatori Computer Center}

The map combination results reported in this section are based on data obtained on the second floor of the USC Salvatori Computer Center, where a complete loop corresponds to approximately 80 meters of traveled distance. As summarized in Table 4.1, a total of 26 map combination experiments were computed offline, combining various pairs from a total set of 19 available maps, $D_{1}$ through $D_{19}$. Some of these combinations involved map pairs built by the same robot at different runs as well, which does not restrict the algorithm's generality.

The third and fourth columns of Table 4.1 indicate the original size of the search space, and its reduced value after applying heuristics $\mathbf{H 1}$ and H2. For comparison, these two numbers, and their relatively stable ratio are plotted 


\begin{tabular}{|c|c|c|c|c|c|}
\hline $\begin{array}{c}\text { Combined } \\
\text { Data Sets }\end{array}$ & of Travel & $\begin{array}{c}\text { Total } \\
\text { Number of } \\
\text { Candidates }\end{array}$ & $\begin{array}{c}\text { Number of } \\
\text { Selected } \\
\text { Candidates }\end{array}$ & $\begin{array}{c}\text { Correctness } \\
\text { of the Match } \\
\text { (\%) }\end{array}$ & $\begin{array}{c}\text { Eliminated } \\
\text { False Alarm } \\
\text { (\%) }\end{array}$ \\
\hline$D_{1}-D_{2}$ & same & 15 & 4 & 100 & - \\
\hline$D_{15}-D_{16}$ & opposite & 15 & 4 & 100 & - \\
\hline$D_{3}-D_{4}$ & same & 30 & 8 & 100 & - \\
\hline$D_{16}-D_{17}$ & same & 33 & 6 & 100 & 100 \\
\hline$D_{3}-D_{5}$ & same & 40 & 11 & 100 & 100 \\
\hline$D_{4}-D_{5}$ & same & 48 & 8 & 100 & 100 \\
\hline$D_{1}-D_{6}$ & opposite & 51 & 4 & 100 & - \\
\hline$D_{1}-D_{7}$ & opposite & 54 & 5 & 100 & - \\
\hline$D_{15}-D_{16}$ & opposite & 55 & 11 & 100 & 100 \\
\hline$D_{1}-D_{8}$ & same & 60 & 5 & 100 & 100 \\
\hline$D_{1}-D_{9}$ & opposite & 60 & 6 & 100 & 100 \\
\hline$D_{10}-D_{11}$ & same & 65 & 9 & 50 & 50 \\
\hline$D_{11}-D_{12}$ & opposite & 70 & 7 & 100 & - \\
\hline$D_{12}-D_{13}$ & same & 70 & 10 & 100 & 100 \\
\hline$D_{2}-D_{6}$ & opposite & 85 & 12 & 75 & - \\
\hline$D_{18}-D_{19}$ & opposite & 88 & 6 & 86 & 80 \\
\hline$D_{2}-D_{7}$ & opposite & 90 & 15 & 100 & - \\
\hline$D_{12}-D_{14}$ & opposite & 91 & 9 & 100 & 100 \\
\hline$D_{2}-D_{8}$ & same & 100 & 13 & 60 & 50 \\
\hline$D_{2}-D_{9}$ & opposite & 100 & 16 & 100 & 100 \\
\hline$D_{11}-D_{13}$ & opposite & 100 & 11 & 100 & 100 \\
\hline$D_{12}-D_{13}$ & opposite & 130 & 12 & 100 & - \\
\hline$D_{6}-D_{7}$ & same & 306 & 20 & 92 & 0 \\
\hline$D_{6}-D_{9}$ & same & 340 & 21 & 100 & 33 \\
\hline$D_{7}-D_{9}$ & same & 360 & 25 & 92 & 66 \\
\hline$D_{8}-D_{9}$ & opposite & 400 & 22 & 70 & 33 \\
\hline Tale & & & & \\
\hline
\end{tabular}

Table 1.26 experiments of map combination.

in Figure 2.

The column indicating the Correctness of the Match is the ratio of correct matches to all reported matches. Finally, the last column gives a measure of the false alarms that were successfully eliminated by combining the maps built by different robots (or, by the same robot at different times). Since the probability of both robots experiencing the same false positive at the same location is lower than it is in the single robot case, multi-robot mapping is inherently well-suited to eradicating false positives (e.g., the feature detector can mistakenly signal the presence of a door due to noisy sensory input).

The first example of map combination is depicted on Figures 3 through 4. At a first glance, the maps $D_{9}$ and $D_{7}$ may seem to have most landmarks of the building in common. Yet, a closer look reveals that in $D_{9}$, the robot explored a cul-de-sac in the lower right corner of the map, which does not 

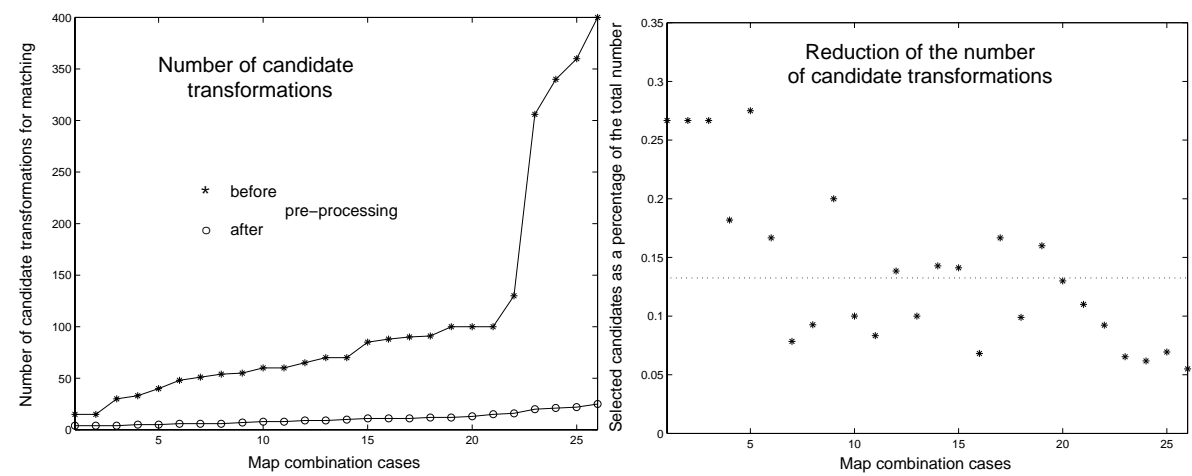

Fig. 2. For all map combination cases summarized in Table 4.1, the heuristics reduce the number of transformation candidates drastically (left), also plotted as a percentage of the initial set (right).

exist in $D_{7}$. Furthermore, their sets of detected doors do not perfectly overlap. Despite all the discrepancies, the matching process was able to collect enough evidence for the transformation correctly proposed in Figure 4.

A more interesting example of map matching involves $D_{1}$, which is an unfinished map as shown in Figure 5 (left). In $D_{1}$, the robot has only had a chance to explore the northern corridor of the same area as in $D_{9}$, but traveling in the opposite direction. As can be seen in Figure 5, $D_{1}$ and $D_{9}$ are still matched successfully. From the robot's point of view, this allows time-critical localization decisions to be made with respect to other robots or a global map.
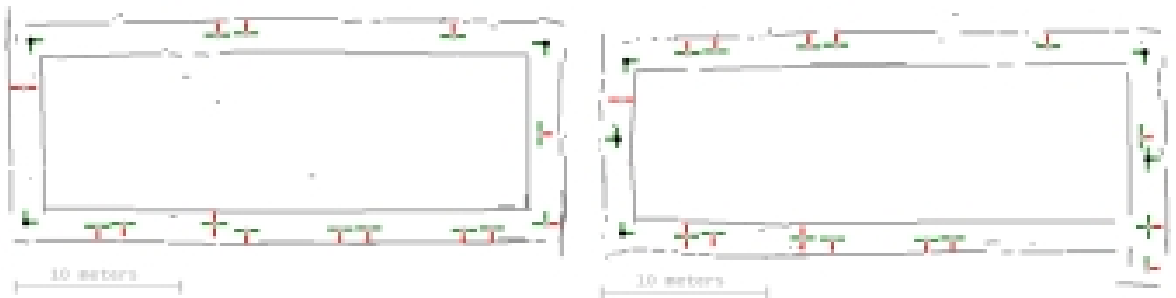

Fig. 3. Map $D_{9}$ (left) of the Salvatori Center. The robot traveled in a counterclockwise direction, registering doors and junctions as they are detected. In an independent mapping experiment, the robot detected a slightly different set of landmarks as shown in map $D_{7}$ (right). 


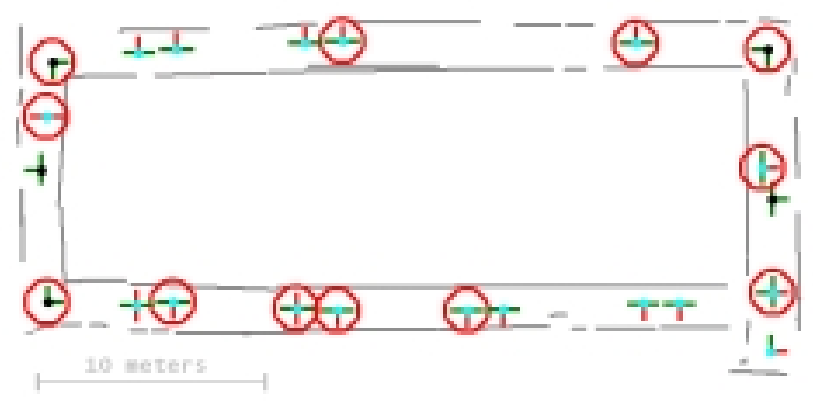

Fig. 4. Maps $D_{7}$ and $D_{9}$ of the same environment are matched against each other and their combination is correctly generated. Circles indicate features that were registered in both maps.

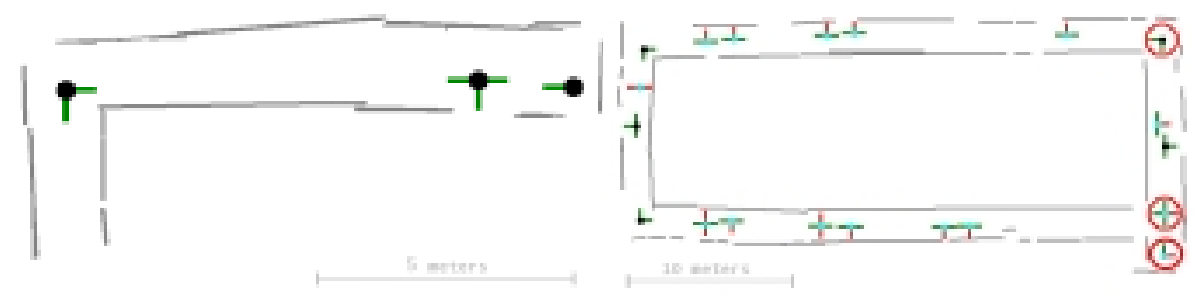

Fig. 5. A snapshot of the map built by Milka ( $D_{1}$, left), which has so far only explored the northern corridor of the Salvatori Center, navigating in a clockwise direction. Salvatori maps $D_{1}$ and $D_{9}$ are correctly combined (right).

\subsection{Preliminary on-line multi-robot trials}

Our matching algorithm was also tested on-line with robots building maps in parallel and independently from each other. The data included here is a representative case of many multi-robot mapping experiments performed during the DARPA Tactical Mobile Robotics experiments in July 1999, which took place in an empty hospital building in Fort Sam Houston, San Antonio. Figure 6 shows full-screen snapshots of the maps built by Vespucci and Milka. In this experiment, Vespucci first explored the West wing of the hospital building, and then made a tour around the central elevator block. On the other hand, Milka started its journey at the East wing, also followed by a tour around the elevator block. Figure 7 shows how their maps were combined. Based on the evidence that both robots collected while turning around the central block, they were cooperatively able to produce a complete map of the scene. 
Finally, a short remark about the metric accuracy of the maps is in order. In Figure 8, the combined map of Figure 7 was overlaid on the floor plan of the hospital. Over a stretch of more than 50 meters of corridor, all mapped wall segments were within 1 meter of their corresponding position according to the blue prints. This rough measure indicates a metric accuracy of about $2 \%$ of our mapping scheme.

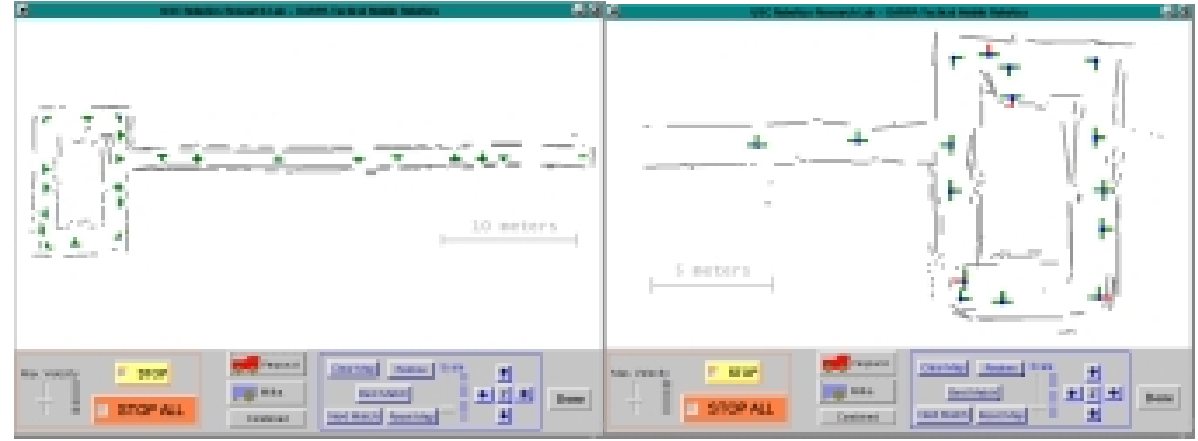

Fig. 6. Map built by Vespucci (left), starting from the West wing of the building. The second map was built by Milka (right), which started from the East wing of the building.

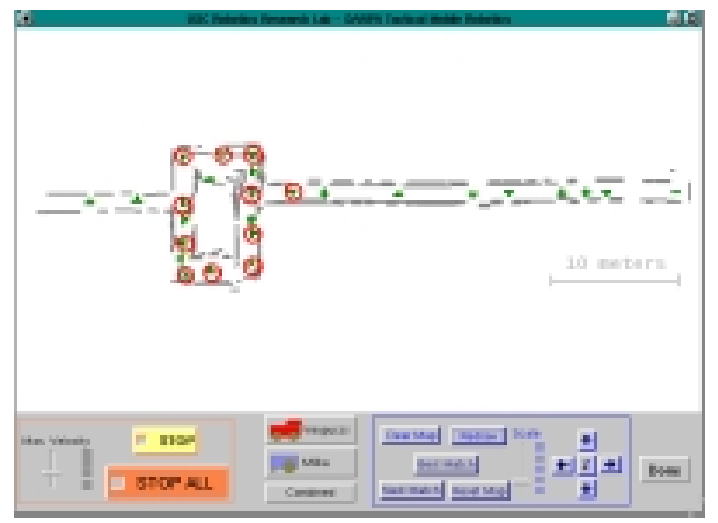

Fig. 7. Combined maps of Vespucci and Milka provides the complete picture that was not available to individual robots. 


\section{Conclusions}

We have proposed and evaluated a map matching algorithm that allows mobile robots to combine their augmented topological maps with those of others. Extensive offline tests of the algorithm suggest that the approach will efficiently scale to the multi-robot case, and also provide a reasonably cheap framework for a group of robots to localize themselves with respect to each other. Preliminary results from the first online, two-robot implementation are also presented.

The extension of this algorithm to the multi-robot case is currently underway. Future work on the mapping scheme will include the use of probability distributions for feature sets. For the multi-robot case, a generic version of the algorithm will be made part of the existing mapping system so that multiple robots can dynamically combine their maps to produce a unified representation of their common surroundings.

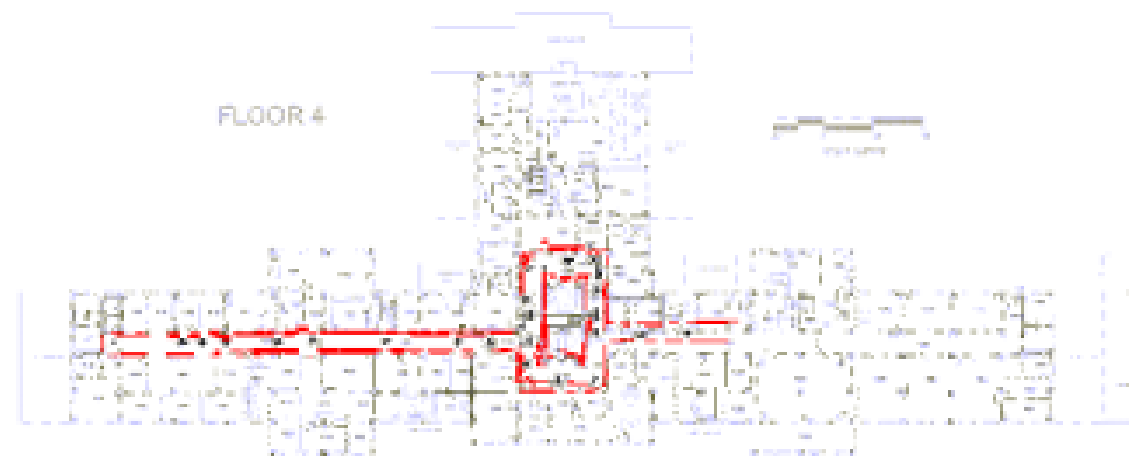

Fig. 8. Combined maps laid over the 4th floor plan of the hospital building.

\section{Acknowledgments}

The authors would like to thank Kale Harbick for hardware and operating system support, and Richard Vaughan for helping with demonstrations. This work is supported by contracts F04701-97-C-0021 and DAAE07-98-C-L028 from DARPA under the TMR program, and by grant DABT63-99-1-0015 from DARPA under the MARS program.

\section{References}

1. G. Beccari, S. Caselli, and F. Zanichelli. Qualitative spatial representations from task-oriented perception and exploratory behaviors. Robotics and $\mathrm{Au}$ tonomous Systems, 25:147-157, 1998. 
2. J. Borenstein, B. Everett, and L. Feng. Navigating Mobile Robots: Systems and Techniques. A. K. Peters Ltd., Wellesley, MA, 1996.

3. Anthony R. Cassandra, Leslie Pack Kaelbling, and James A. Kurien. Acting under uncertainty: Discrete bayesian models for mobile robot navigation. In Proceedings of IEEE/RSJ International Conference on Intelligent Robots and Systems, 1996.

4. Howie Choset. Sensor Based Motion Planning: The Hierarchical Generalized Voronoi Graph. PhD thesis, California Institute of Technology, 1996.

5. Ingemar J. Cox. Blanche - an experiment in guidance and navigation of an autonomous robot vehicle. IEEE Transactions on Robotics and Automation, 7(2):193-204, April 1991.

6. Göksel Dedeoglu, Maja J. Mataric, and Gaurav S. Sukhatme. Incremental, online topological map building with a mobile robot. In Proceedings of the Photonics East Conference, Boston, MA, 1999. SPIE.

7. Alberto Elfes. Occupancy grids: A Probabilistic framework for robot perception and navigation. $\mathrm{PhD}$ thesis, Carnegie Mellon University, 1989.

8. Benjamin Kuipers and Yung-Tai Byun. A robot exploration and mapping strategy based on a semantic hierarchy of spatial representations. Robotics and Autonomous Systems, 8:47-63, 1991.

9. Maja J. Mataric. Integration of representation into goal-driven behavior-based robots. IEEE Transactions on Robotics and Automation, 8(3):304-312, 1992.

10. Hans P. Moravec and Alberto Elfes. High resolution maps from wide angle sonar. In Proceedings of the IEEE International Conference on Robotics and Automation, pages 116-121, St. Louis, Missouri, 1985.

11. Hagit Shatkay and Leslie Pack Kaelbling. Learning topological maps with weak local odometric information. In Proceedings of the 15th International Joint Conference on Artificial Intelligence (IJCAI-97), pages 920-929, San Francisco, August 23-29 1997. Morgan Kaufmann Publishers.

12. Reid Simmons and Sven Koenig. Probabilistic robot navigation in partially observable environments. In Proceedings of the International Joint Conference on Artificial Intelligence, pages 1080-1087, 1995.

13. Sebastian Thrun, W. Burgard, and Dieter Fox. A probabilistic approach to concurrent mapping and localization for mobile robots. Machine Learning and Autonomous Robots (joint issue), 31/5:29-53 / 253-271, 1998.

14. B. Yamauchi, A. C. Schultz, and W. Adams. Mobile robot exploration and map-building with continuous localization. In Proceedings of the IEEE International Conference on Robotics and Automation (ICRA-98), pages 3715-3720, Piscataway, May 16-20 1998. IEEE Computer Society. 